\title{
Midterm outcomes after postoperative delirium on cognition and mood in patients after cardiac surgery
}

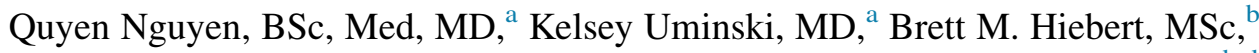 \\ Navdeep Tangri, MD, PhD, FRCPC, ${ }^{c}$ and Rakesh C. Arora, MD, PhD, FRCSC ${ }^{\mathrm{b}, \mathrm{d}}$
}

\section{ABSTRACT}

Objective: Delirium is a common neurologic complication after cardiac surgery. Our primary objective was to determine the impact of delirium on self-reported problems with midterm cognitive functioning and mood postcardiac surgery.

Methods: A single-center, prospective cohort study was conducted, enrolling 197 patients undergoing coronary artery bypass grafting or valve replacement. Baseline cognition and mood were assessed preoperatively in elective patients as a part of routine care using the Montreal Cognitive Assessment and Patient Health Questionnaire 9, respectively. During hospitalization, delirium was diagnosed using the Confusion Assessment Method (Confusion Assessment Method/Confusion Assessment Method Intensive Care Unit). Follow-up assessments were carried out via telephone interview at 6 to 9 months after cardiac surgery using the EuroQoL-5D, Patient Health Questionnaire 9, Alcohol Use Disorders Identification Test Consumption, Memory Impairment Screen, and Category Fluency Test assessments. We examined resultant scores in the domains of cognition and mood among delirious and nondelirious cohorts, controlling for confounders deemed clinically relevant.

Results: A total of 197 patients were enrolled in the study, of whom $44(22 \%)$ developed postoperative delirium. After adjustment, no differences were observed in cognitive functioning between the delirious and nondelirious subjects after 6 to 9 months. However, delirious patients were more likely to experience selfreported problems in affective (depression and anxiety) functioning as measured by the EuroQoL-5D (odds ratio, 4.41; 95\% confidence interval, 1.51-12.92; $P<.01)$.

Conclusions: Postoperative delirium is associated with increased anxiety and depression at 6 to 9 months postcardiac surgery. Future investigation should seek to evaluate the utility of screening programs for affective disorders in those individuals who develop delirium in the postoperative period. (J Thorac Cardiovasc Surg 2018;155:660-7)

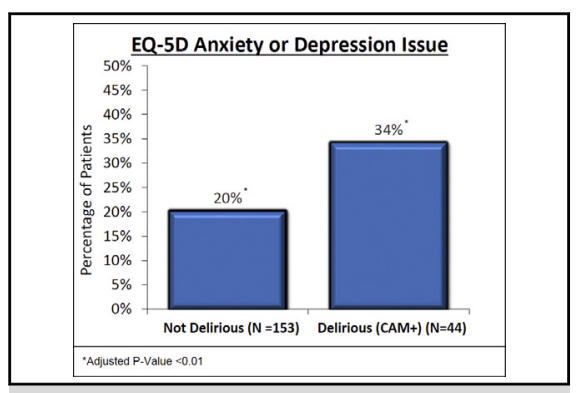

Delirium places patients at increased risk for anxiety and depression after surgery.

\section{Central Message}

Postoperative delirium is common after cardiac surgery, placing patients at risk for subsequent anxiety and depression at midterm follow-up.

\section{Perspective}

Given the incidence of postoperative delirium among patients undergoing cardiac surgery and the risk for subsequent anxiety and depression, clinicians must consider ways to improve the health of this population. Various perioperative predictors identified to be associated with postoperative delirium may lend themselves to screening programs for affective disorders in these vulnerable individuals.

See Editorial Commentary page 668.

See Editorial page 657.
From the a Max Rady College of Medicine, Rady Faculty of Health Sciences, Departments of ${ }^{\mathrm{c}}$ Internal Medicine, and ${ }^{\mathrm{d}}$ Surgery, University of Manitoba, Manitoba; and ${ }^{\mathrm{b}}$ Cardiac Sciences Program, Winnipeg Regional Health Authority, Winnipeg, Canada.

N.T. and R.C.A. have received an operating grant from the Department of Surgery, Max Rady College of Medicine, University of Manitoba, for this work.

Received for publication Sept 22, 2016; revisions received Sept 16, 2017; accepted for publication Sept 28, 2017; available ahead of print Nov 10, 2017.

Address for reprints: Rakesh C. Arora, MD, PhD, FRCSC, St Boniface Research Centre/I.H. Asper Clinical Research Institute, University of Manitoba, Winnipeg, Manitoba, Canada R2H 2A6 (E-mail: rakeshcarora@gmail.com).

$0022-5223 / \$ 36.00$

Copyright (c) 2017 by The American Association for Thoracic Surgery

https://doi.org/10.1016/j.jtcvs.2017.09.131
Delirium is an acute confusional state characterized by inattention, disorganized thinking, and altered, often fluctuating levels of consciousness. ${ }^{1}$ It remains a common and increasingly prevalent neurologic complication after cardiac

Uf Scanning this QR code will take
you to supplemental tables for
this article.




\section{Abbreviations and Acronyms \\ AUDIT-C = Alcohol Use Disorders Identification Test Consumption \\ CAM $=$ Confusion Assessment Method \\ CAM-ICU $=$ Confusion Assessment Method for the ICU \\ CFT $=$ Category Fluency Test \\ EQ-5D $=$ EuroQoL-5D \\ euroSCORE $=$ European System for Cardiac \\ Operative Risk Evaluation \\ MIS $=$ Memory Impairment Screen \\ $\mathrm{MoCA}=$ Montreal Cognitive Assessment \\ OR = odds ratio \\ PHQ-9 = Patient Health Questionnaire 9 \\ RASS $\quad=$ Richmond Agitation-Sedation Scale}

surgery, with current estimates of at least 1 in 7 patients affected. $^{2,3}$ As the risk profile of surgical candidates continues to change with the inclusion of more extensive cardiovascular disease and medical comorbidities, the incidence of postoperative delirium is expected to increase.

Increasing attention has been paid to the negative effects of delirium on patient health outcomes, which include loss of patient independence, increased morbidity, and increased short- and long-term mortality. ${ }^{4,5}$ Cumulatively, the financial burden of delirium on healthcare systems is staggering. ${ }^{6}$ Although considered independent risk factors ${ }^{7}$ for the occurrence of postoperative delirium, cognitive and affective functional decline may occur secondary to delirium in some patients, ${ }^{8-11}$ challenging persistent beliefs that acute changes in delirium are indeed transitory and reversible.

To date, a relationship between postoperative delirium and both cognitive impairment and affective functioning in the setting of cardiac surgery has not been extensively investigated. $^{9-11}$ Therefore, the purpose of our study was (1) to evaluate the effect of postoperative delirium on midterm (69 months) cognition in patients after cardiac surgery; (2) to evaluate the effect of postoperative delirium on mental health, including anxiety and depression, after cardiac surgery; and (3) to determine risk factors for each of these conditions.

\section{MATERIALS AND METHODS Study Population}

A prospective observational cohort study was carried out at the St Boniface Hospital, a tertiary care center in Winnipeg, Manitoba, with a patient catchment area of approximately 1.1 million people. The study was approved by the University of Manitoba Research Ethics Board and the St Boniface Hospital Research Review Committee Research Ethics Board number HS16100 (H2013:090). Patients who were aged more than 18 years, who were undergoing an elective cardiac surgery procedure, who were admitted to the intensive care cardiac surgery unit, and who attended a preoperative clinic were eligible to participate in this study.
Patients in whom postoperative delirium could not reliably be tested (ie, because of previous debilitating stroke, cerebral palsy, severe dementia, severe hearing disabilities, or inability to understand English or French, active seizure disorder, or Child-Pugh class B or C cirrhosis) were excluded from the study. Patient recruitment was limited to daytime hours Monday through Friday. Consecutively consenting patients were recruited from July 16 , 2013, to July 15,2014 , with cardiac surgery dates from February 5, 2013, to February 25, 2014.

\section{Perioperative Care}

All patients undergoing cardiac surgery received a general anesthetic maintained with isoflurane. Sufentanil is the opioid of choice for induction. All patients undergo cardiopulmonary bypass. Approximately $40 \%$ to $60 \%$ of all patients are extubated in the operating room. On arrival to the intensive care cardiac surgery unit, all patients receive intermittent intravenous fentanyl, oral hydromorphone, and acetaminophen titrated for adequate postoperative analgesia. No routine benzodiazepines are used for sedation purposes of intubated patients, with propofol being the agent of choice. In addition, we encourage early ambulation, discourage daytime sleep, and offer melatonin at night for sleep.

\section{Measurement and Outcomes}

Preoperative, intraoperative, and postoperative parameters were extracted for consented patients from the Manitoba Cardiac Surgery Database and the patient's medical records. Preoperative data included patient demographics, surgical risk scores (European System for Cardiac Operative Risk Evaluation [euroSCORE] $\mathrm{II}^{12}$ ), medical history, cardiac risk factors, and cardiac illness severity. Baseline cognitive functioning and mood were assessed as a part of routine care using the Montreal Cognitive Assessment $(\mathrm{MoCA})^{13}$ and Patient Health Questionnaire-9 (PHQ-9) ${ }^{14}$ respectively, for elective patients during their visit to the Cardiac Preoperative Assessment Clinic or preoperatively on the inpatient ward (ie, "in-house urgent" patients). Perioperative data included intraoperative anesthesia, cardiopulmonary bypass details, return to operating room because of bleeding, acute kidney injury, and cerebrovascular accident. In addition to collection of new postoperative delirium, postoperative data included intensive care unit (ICU) and hospital length of stay, 28-day inhospital mortality, and major adverse events (including death, myocardial infarction, stroke, and renal failure requiring dialysis).

Patients were assessed for delirium routinely every 4 hours by bedside RN personnel while in the ICU using the Confusion Assessment Method for the ICU (CAM-ICU) ${ }^{15}$ and every 8 hours on the hospital wards using the Confusion Assessment Method (CAM). ${ }^{16}$ All personnel underwent formal education on the CAM-ICU and CAM tools using previously described implementation methodologies. ${ }^{17,18}$ Episodes of delirium were collected up to postoperative day 10. Patients were classified as having a delirious day if delirium was detected in at least 1 assessment during the 24-hour period of a given postoperative day. Patients were contacted and consented via telephone 6 to 9 months postcardiac surgery and underwent a telephone interview using standardized health surveys chosen to assess cognition, anxiety, depression, and substance abuse.

Affective functioning and substance abuse were evaluated 6 to 9 months after cardiac surgery through administration of the EuroQol 5D (EQ5D) ${ }^{19,20}$ PHQ-9, ${ }^{14}$ and Alcohol Use Disorders Identification Test Consumption (AUDIT-C) ${ }^{21}$ assessments. The PHQ-9 has been validated in various studies and incorporates the Diagnostic and Statistical Manual of Mental Disorders diagnostic criteria for depression. Depression from the PHQ-9 score was defined as a score of 5 or higher. The EQ-5D is a validated measures of health-related quality of life and was selected for its simplicity and proven clinical relevance. ${ }^{19}$ An individual was defined as having an "issue" with a given component of the EQ-5D if he/she reported a score of greater than 1. Furthermore, the AUDIT-C is a widely used, 3-item questionnaire on alcohol consumption habits. Patient subjective responses of having mild problems or worse were considered significant. 
Midterm cognition was assessed using the Memory Impairment Screen $(\mathrm{MIS})^{22,23}$ and the Category Fluency Test (CFT). ${ }^{24}$ The MIS has been validated as an efficient, reliable, and valid screening for dementia and Alzheimer's disease, and the CFT has been validated and used in studies of Alzheimer's disease in both literate and illiterate patients. An individual was defined as being cognitively impaired on the basis of the MIS criteria if they scored 4 or less. Cognitive impairment based on the CFT screen was defined as a score of 12 or less. Both of the cognition assessment scores from the MIS and CFT were compared directly between the delirious and nondelirious patient groups, and were adjusted for confounders such as baseline MoCA.

\section{Outcomes and Statistical Analysis}

Several preoperative, intraoperative, and postoperative characteristics of the study cohort were compared between delirious and nondelirious patients. Continuous variables were compared using the Mann-Whitney test. Categoric variables were compared using the chi-square test or Fisher exact test. The primary study outcomes of 6- to 9-month cognition and affective functioning were explored using logistic regression. An unadjusted model with in-hospital delirium as the lone predictor was run for each of the primary outcomes. In addition, 2 adjusted models were run for each outcome in an attempt to control for relevant confounders while providing an adjusted odds ratio (OR) and corresponding 95\% confidence interval (CI) and $P$ value for in-hospital delirium. These models controlled for risk factors that were deemed clinically relevant by the research team after review of previous literature. The first adjusted model controlled for age, sex, and the preoperative risk of mortality via the euroSCORE II. The second model adjusted for age, sex, and euroSCORE II, and the baseline MoCA score for the cognition screening outcomes and the baseline PHQ-9 score for the mood screening outcomes. All analyses were performed using SAS version 9.3 (SAS Institute Inc, Cary, NC).

\section{RESULTS}

\section{Prevalence of Delirium}

Of the 371 patients approached, 199 (53.6\%) consented to participate in the study. The remaining 172 patients did not consent, were unable to be contacted after 3 separate attempts, or did not meet inclusion criteria (Figure 1). Of those who consented to study participation, 197 patients were successfully linked to the Manitoba Cardiac Surgical Database (ie, 2 patients could not be linked to the provincial database) and served as the study cohort. A total of 171 patients $(86.8 \%)$ were elective surgical patients, and the remaining 26 patients $(13.2 \%)$ were in-house urgent surgical patients. Within the elective surgical population, 36 patients $(21.1 \%)$ were delirious in the postoperative period $(\mathrm{CAM}+)$, whereas 8 patients $(30.8 \%)$ in the inhouse urgent surgical group were affected. On the basis of their Richmond Agitation-Sedation Scale (RASS) score(s), of the 44 patients who were diagnosed with postoperative delirium, 32 (73\%) were of the hypoactive subtype (RASS $\leq 0), 1(2 \%)$ was of the hyperactive subtype (RASS $>0)$, and $11(25 \%)$ experienced a mixed episode (RASS $\leq 0$ and RASS $>0$ ).

\section{Factors Associated With Postoperative Delirium}

Patients with delirium were older (72.6 vs 68.7 years, $P<.01)$, had a higher euroSCORE II (3.1 vs 1.6, $P<.01$ ), were less likely to have a high school education $(54.6 \%$ vs $34 \%, P=.01)$, were more likely to have a lower ejection fraction $(18.9 \%$ vs $6.3 \%, P=.04)$, and were more likely to have undergone a previous cardiovascular procedure $(25 \%$ vs $7.4 \%, P<.01)$ (Table 1$)$. No other significant differences in preoperative characteristics between delirious and nondelirious patients were observed.

Patients who experienced other postoperative complications were also at a higher risk of postoperative delirium. In particular, a return to the operative room for bleeding $(P<.01)$, acute kidney injury $(P<.01)$, and cerebrovascular accident $(P<.01)$

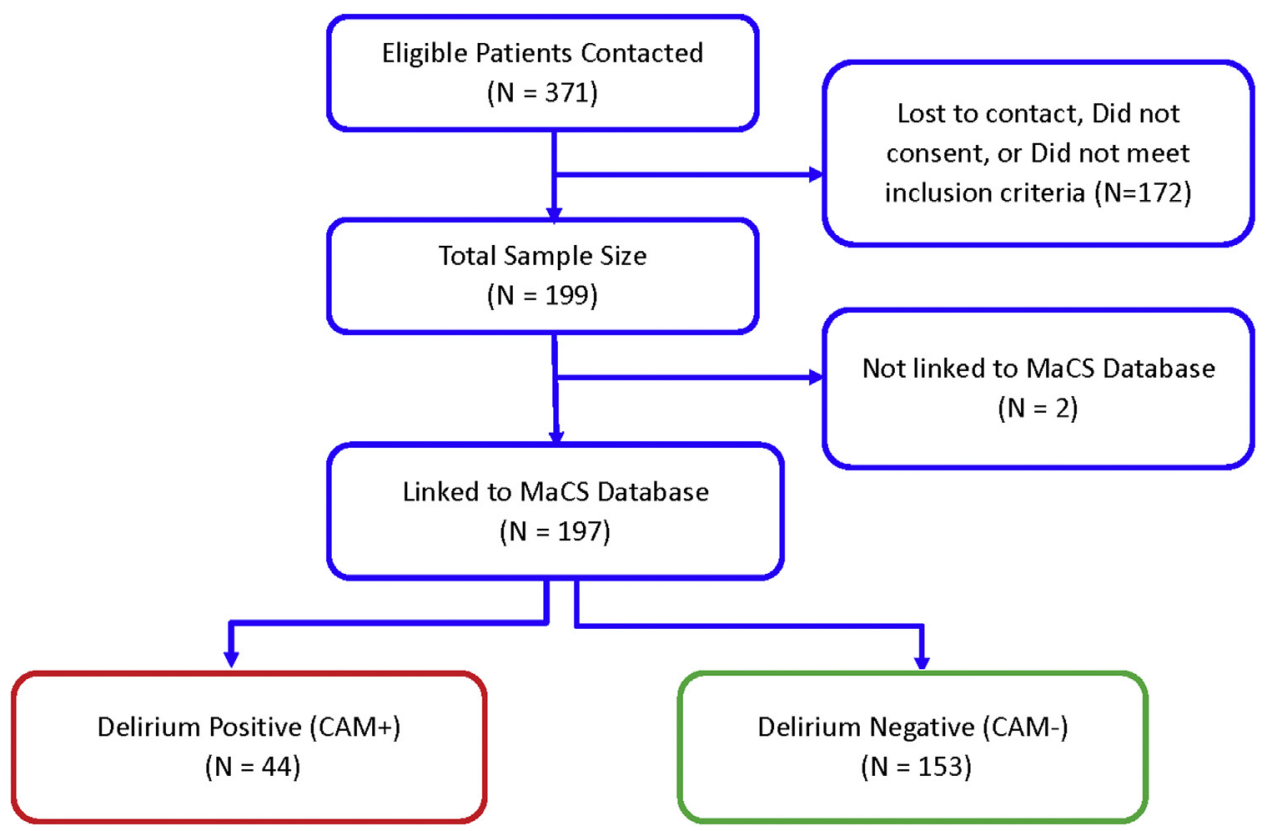

FIGURE 1. Sample size breakdown for study cohort. MaCS, Manitoba Cardiac Surgery Database; CAM, Confusion Assessment Method. 
TABLE 1. Association between preoperative patient characteristics and postoperative delirium $(\mathrm{CAM}+)$

\begin{tabular}{|c|c|c|c|c|}
\hline Variable & Full cohort $(N=197)$ & Delirious $(N=44)$ & Not delirious $(N=153)$ & $P$ value \\
\hline Age, $y$ & $69.9(60.7-76.2)$ & $72.6(67.8-79.6)$ & $68.7(59.4-75.1)$ & $<.01$ \\
\hline Gender (female) & $60(30.5 \%)$ & $12(27.3 \%)$ & $48(31.4 \%)$ & .60 \\
\hline BMI, $\mathrm{kg} / \mathrm{m}^{2}$ & $28.7(25.8-32.1)$ & $29.1(24.7-33.5)$ & $28.6(25.9-31.6)$ & .50 \\
\hline Urgent case & $26(13.2 \%)$ & $8(18.2 \%)$ & $18(11.8 \%)$ & .30 \\
\hline Lives alone & $36(18.3 \%)$ & $6(13.6 \%)$ & $30(19.6 \%)$ & .30 \\
\hline Less than high school education & $76(38.6 \%)$ & $24(54.6 \%)$ & $52(34.0 \%)$ & .01 \\
\hline White & $174(88.2 \%)$ & $37(84.1 \%)$ & $137(89.5 \%)$ & .30 \\
\hline Hypertension & $154(78.2 \%)$ & $34(77.3 \%)$ & $120(78.4 \%)$ & .90 \\
\hline MI & $33(16.8 \%)$ & $6(13.6 \%)$ & $27(17.7 \%)$ & .50 \\
\hline Diabetes & $50(25.4 \%)$ & $13(29.6 \%)$ & $37(24.2 \%)$ & .50 \\
\hline Renal insufficiency $(\mathrm{Cr}>176)$ & $4(2.0 \%)$ & $1(2.3 \%)$ & $3(2.0 \%)$ & 1.00 \\
\hline Dialysis & $2(1.0 \%)$ & $1(2.3 \%)$ & $1(0.7 \%)$ & .40 \\
\hline Congestive heart failure & $29(14.7 \%)$ & $6(13.6 \%)$ & $23(15.1 \%)$ & .80 \\
\hline COPD & $19(9.6 \%)$ & $2(4.6 \%)$ & $17(11.1 \%)$ & .30 \\
\hline CVA & $7(3.6 \%)$ & $3(6.8 \%)$ & $4(2.6 \%)$ & .20 \\
\hline Atrial fibrillation & $28(14.2 \%)$ & $6(13.6 \%)$ & $22(14.4 \%)$ & .90 \\
\hline Ejection fraction $<35 \%$ & $15(9.1 \%)$ & $7(18.9 \%)$ & $8(6.3 \%)$ & .04 \\
\hline Previous cardiovascular procedure & $22(11.4 \%)$ & $11(25.0 \%)$ & $11(7.4 \%)$ & $<.01$ \\
\hline PHQ-9 baseline* & $2.0(1.0-3.0)$ & $1.5(1.0-3.0)$ & $2.0(1.0-3.0)$ & .95 \\
\hline PHQ-9 status (depressed)* & $25(16.0 \%)$ & $6(17.7 \%)$ & $19(15.6 \%)$ & .80 \\
\hline MoCA score-baseline* & $25.0(23.0-28.0)$ & $24.5(20.0-27.0)$ & $25.0(23.0-28.0)$ & .07 \\
\hline euroSCORE II (\%) & $1.6(1.1-3.3)$ & $3.1(1.5-8.1)$ & $1.6(1.0-2.7)$ & $<.01$ \\
\hline
\end{tabular}

Continuous variables are expressed as median (interquartile range) and compared using Mann-Whitney test. Categoric variables are expressed as N (\%) and compared using chisquare test or Fisher exact test. BMI, Body mass index; $M I$, myocardial infarction; $C r$, creatinine; $C O P D$, chronic obstructive pulmonary disease; $C V A$, cardiovascular accident; PHQ-9, Patient Health Questionnaire 9; MoCA, Montreal Cognitive Assessment; euroSCORE, European System for Cardiac Operative Risk Evaluation. *Variable was collected for elective patients only $(\mathrm{N}=171)$.

were all associated with an increased risk of postoperative delirium (Table 2). In addition, intraoperative and postoperative blood transfusions $(P<.01)$, and longer cardiopulmonary bypass times $(P=.02)$ also appeared to be associated with postoperative delirium. Delirious patients remained in the ICU approximately 4 times longer ( 81.1 vs 23.2 hours, $P<.01)$ than nondelirious patients and were hospitalized 5 days longer (12 vs 7 days, $P<.01)$ (Table 3 ).

\section{Postoperative Delirium and Cognition}

Compared with nondelirious patients, patients who experienced postoperative delirium obtained lower scores on the MIS (OR, 3.15; 95\% CI, 140-7.09; $P<.01)$. No difference in scores for the CFT was noted (OR, 1.60; 95\% CI, 0.73$3.50 ; P<.24)$. However, adjusting for patient age, sex, euroSCORE II, and baseline MoCA, differences in MIS scores were no longer significant, whereas differences in CFT scores remained similar in magnitude and overall direction (Table 4 and Tables E1-E3).

\section{Postoperative Delirium and Mood}

Patients who had been delirious after surgery did not experience more self-reported problems in mood or alcohol consumption (Table 5), as reflected in the unadjusted EQ-5D (OR, 2.04; 95\% CI, 0.97-4.26; $P<.0589$ ), PHQ-9 (OR, 1.19; $95 \% \mathrm{CI}, 0.57-2.49 ; P<.654)$, and AUDIT-C scores (OR, 0.54; 95\% CI, 0.24-1.21; $P<.1354$ ). However, when accounting for patient age, sex, euroSCORE II, and baseline PHQ-9, differences in self-reported mood, as reflected by the EQ-5D score, were significant (OR, 4.41; 95\% CI, 1.5112.92; $P<.0068)$. The remaining scores for PHQ-9 and AUDIT-C remained insignificant after adjustments.

\section{DISCUSSION}

In our prospective cohort study of patients undergoing cardiac surgery, we found that postoperative delirium was associated with increased anxiety and depression at 6 to 9 months postcardiac surgery. In contrast, no difference in cognitive functioning or alcohol consumption was detected between the delirious and nondelirious groups. The overall incidence of delirium in this cohort was $21.1 \%$ and $30.8 \%$ in elective and emergency surgical populations, respectively. Together, these findings confirm that postoperative delirium is a common neurologic complication that places patients at increased risk for affective disorders after hospital discharge. 
TABLE 2. Association between intraoperative and postoperative patient characteristics and postoperative delirium (CAM + )

\begin{tabular}{|c|c|c|c|c|}
\hline Variable & Full cohort $(N=197)$ & Delirious $(N=44)$ & Not delirious $(\mathbf{N}=153)$ & $P$ value \\
\hline Type of procedure & & & & .40 \\
\hline CABG & $69(35.0 \%)$ & $13(29.6 \%)$ & $56(36.6 \%)$ & \\
\hline Valve & $67(34.0 \%)$ & $14(31.8 \%)$ & $53(34.6 \%)$ & \\
\hline $\mathrm{CABG}+$ valve & $26(13.2 \%)$ & $9(20.5 \%)$ & $17(11.1 \%)$ & \\
\hline Other & $35(17.8 \%)$ & $8(18.2 \%)$ & $27(17.7 \%)$ & \\
\hline Cardiopulmonary bypass time (min) & $103(80-140)$ & $116(83-184)$ & $100(79-128)$ & .02 \\
\hline Intraoperative transfusion (red blood, plasma, or platelets) & $77(39.1 \%)$ & $25(56.8 \%)$ & $52(34.0 \%)$ & $<.01$ \\
\hline Postoperative transfusion (red blood, plasma, or platelets) & $87(44.2 \%)$ & $31(70.5 \%)$ & $56(36.6 \%)$ & $<.01$ \\
\hline Return to operating room, bleeding & $14(7.1 \%)$ & $8(18.2 \%)$ & $6(3.9 \%)$ & $<.01$ \\
\hline Acute kidney injury $(<50 \%$ increase in $\mathrm{Cr}$ ) & $16(8.5 \%)$ & $9(20.9 \%)$ & $7(4.8 \%)$ & $<.01$ \\
\hline New dialysis & $8(4.1 \%)$ & $4(9.1 \%)$ & $4(2.6 \%)$ & .08 \\
\hline Pneumonia & $14(7.1 \%)$ & $6(13.6 \%)$ & $8(5.2 \%)$ & .09 \\
\hline Cerebrovascular accident & $5(2.5 \%)$ & $4(9.1 \%)$ & $1(0.7 \%)$ & $<.01$ \\
\hline Cardiac arrest & $5(2.5 \%)$ & $3(6.8 \%)$ & $2(1.3 \%)$ & .07 \\
\hline
\end{tabular}

Continuous variables are expressed as median (interquartile range) and compared using Mann-Whitney test. Categoric variables are expressed as $\mathrm{N}(\%)$ and compared using chisquare test or Fisher exact test. $C A B G$, Coronary artery bypass grafting; $C r$, creatinine.

To our knowledge, our study provides one of the first assessments of midterm cognition and affective functioning using multiple validated questionnaires for each of the evaluated domains, in the context of a systematic delirium assessment program in both the ICU and the postoperative cardiac surgery ward. Mood disorders, notably depression, have been described in the literature as a common occurrence after cardiac surgery, ${ }^{25}$ with some citations reporting 1 in 3 patients affected at time of discharge. ${ }^{26}$ However, some studies examining this clinical question have been hampered by poor methodology and failure to account for baseline mood status before surgery. Therefore, the occurrence of depression and anxiety after cardiac surgery has not been well described or rigorously evaluated in the context of postoperative delirium.

In the literature, the impact of postoperative delirium on cognition has been inconsistent. Studies that have evaluated cognitive function after cardiac surgery varied with respect to the timing and frequency of follow-up assessment. One study ${ }^{10}$ recently assessed cognitive function in 225 patients (incidence of delirium $46 \%$ ) at multiple times points beginning on postoperative day 2 to 1 year after surgery. The findings of this study concluded that delirium was associated with a significant decline in cognitive ability during the first year after cardiac surgery, with a trajectory characterized by an initial decline and prolonged impairment. However, an additional report ${ }^{11}$ assessing cognitive function, in which $17 \%$ developed delirium, concluding memory and concentration deficits among those affected, failed to account for preoperative cognitive function. Although no association between postoperative delirium and cognitive function was noted in our study, we assessed baseline cognitive function in the preoperative period, allowing us to more appropriately characterize cognitive function among patients among delirious and nondelirious patient groups; this is reflected in the loss of statistical significance for MIS scores when accounting for confounders: patient age, sex, euroSCORE II, and baseline MoCA.

In our study, a number of preoperative risk factors for delirium were identified: advanced patient age, high euroSCORE II, lower level of education, and ejection fraction less than $35 \%$. The finding of reduced ejection fraction as an independent predictor of postoperative delirium has been described in the literature. ${ }^{27}$ Parikh and Chung ${ }^{28}$ suggested that older patients have decreased cerebral neuronal density, blood blow, metabolism, and levels of neurotransmitters, which may account for the increased predisposition to postoperative delirium. Of note, the authors

TABLE 3. Intensive care unit and hospital length of stay comparisons between delirious and nondelirious patients

\begin{tabular}{lcccc}
\multicolumn{1}{c}{ Variable } & Full cohort $(\mathbf{N}=\mathbf{1 9 7})$ & Delirious $(\mathbf{N}=\mathbf{4 4})$ & Not delirious $(\mathbf{N}=\mathbf{1 5 3})$ & $\boldsymbol{P}$ value \\
\hline ICU LOS (h) & $25.8(21.5-70.2)$ & $81.1(41.1-165.9)$ & $23.2(20.5-46.3)$ & $<.01$ \\
Hospital LOS (d) & $7.0(6.0-12.0)$ & $12.0(8.0-23.5)$ & $7.0(5.0-9.0)$ & $<.01$ \\
\hline
\end{tabular}

Continuous variables are expressed as median (interquartile range) and compared using Mann-Whitney test. Categoric variables are expressed as $\mathrm{N}(\%)$ and compared using chisquare test or Fisher exact test. ICU, Intensive care unit; $L O S$, length of stay. 
TABLE 4. Association between postoperative delirium (CAM+) and cognition screening tools 6 months after cardiac surgery

\begin{tabular}{|c|c|c|c|c|c|c|c|c|c|}
\hline \multirow[b]{2}{*}{ Variable } & \multicolumn{3}{|c|}{ Unadjusted model } & \multicolumn{3}{|c|}{ Adjusted model* } & \multicolumn{3}{|c|}{ Adjusted model $\dagger$} \\
\hline & $\overline{\text { OR }}$ & $95 \%$ CI & $\overline{P \text { value }}$ & $\overline{\text { OR }}$ & $95 \% \mathrm{CI}$ & $P$ value & $\overline{\text { OR }}$ & $95 \%$ CI & $P$ value \\
\hline MIS cognitive impairment & 3.15 & $1.40-7.09$ & $<.01$ & 1.70 & $0.67-4.33$ & .30 & 1.02 & $0.33-3.16$ & .97 \\
\hline CFT cognitive impairment & 1.60 & $0.73-3.50$ & .20 & 1.38 & $0.58-3.27$ & .40 & 1.14 & $0.41-3.15$ & .80 \\
\hline
\end{tabular}

OR, Odds ratio; $C I$, confidence interval; MIS, Memory Impairment Screen; $C F T$, Category Fluency Test. *Model adjusts for age, sex, and euroSCORE II. †Model adjusts for age, sex, euroSCORE II, and baseline MoCA.

also suggested that preoperative depression, from the basis of aberrant serotonergic and noradrenergic neurotransmitter systems, may account for delirium predisposition. Although delirious patients had a lower baseline MoCA compared with the nondelirious cohort, a significant association was not found, which may be due to our relatively small sample size.

Intraoperative predictors most strongly associated with postoperative delirium in our patient cohort were increased cardiopulmonary pump time, acute kidney injury, returning to the operating room because of postoperative bleeding, and new cerebrovascular accident. Previous literature ${ }^{29}$ indicates that hypoperfusion and microemboli are 2 potential factors in the development of postoperative delirium. In addition, patients with more severe illnesses typically may require more complex surgeries correlating with an increased cardiopulmonary bypass time, increased risk of bleeding, cerebrovascular accident, kidney failure, and ultimately a higher risk of postoperative delirium.

\section{Study Limitations}

We acknowledge several limitations pertaining to our study. First, cognitive impairment and affective functioning were examined through means of telephone interviews. Although this modality of patient interview has gained in popularity and has proved to be valid and reliable in the context of patient assessments, ${ }^{30,31}$ this manner of administration deviates from the initial patient baseline assessment, possibly limiting the comparability of patient outcomes over time. Furthermore, in applying the patient assessment tools, it is worth noting the potential overlap and confounding effects of concurrent illness; for example, there may have been confounding effects of depression on self-reported cognitive symptoms. In addition, there is overlap among the symptom complexes of depression, anxiety, and acute stress reactions, in addition to the somatic symptoms seen after cardiac surgery. Although a number of tools were applied to tease apart the potential contributions of anxiety, depression, and substance use, the presence of acute stress reactions and posttraumatic stress disorders was not characterized. Future studies are planned for a more detailed examination of post-traumatic stress disorder and acute stress reactions in cardiac surgery patients with postoperative delirium. Although the CAM and CAM-ICU are well validated and highly sensitive modalities in the detection of postoperative delirium, aside from the RASS, which is able to identify the subtype of delirium, it is unable to assess for severity of delirium. For the purpose of this study, however, we were mainly interested in the overall prevalence and incidence of delirium irrespective of severity. We were able to identify various preoperative and postoperative characteristics associated with postoperative delirium as part of our study design. We did not further delineate intraoperative risk factors, such as intraoperative use of benzodiazepine, opioid analgesics, sepsis, hypoxia, and alcohol withdrawal, because they have been well reviewed and the primary objective of our study was not to further delineate the risk factors associated with postoperative delirium, but rather to determine the midterm consequence of the postoperative delirium in the cardiac surgery patient after hospital discharge. We acknowledge that we are not directly controlling for procedure type in the final analysis, but we believe that most of the effect that would be due to procedure type is accounted for in the euroSCORE II calculation. Given this was a single-center study, with a relatively small study population and the possibility of selection bias, it may not be possible to draw far-reaching conclusions, and we would recommend repeating the study with a larger sample size. To confirm that the enrolled patient cohort was representative of all cardiac surgery patients at our center, a post hoc analysis was undertaken to compare patient characteristics

TABLE 5. Association between postoperative delirium (CAM+) and mood screening tools 6 months after cardiac surgery

\begin{tabular}{|c|c|c|c|c|c|c|c|c|c|}
\hline \multirow[b]{2}{*}{ Variable } & \multicolumn{3}{|c|}{ Unadjusted model } & \multicolumn{3}{|c|}{ Adjusted model* ${ }^{*}$} & \multicolumn{3}{|c|}{ Adjusted model +} \\
\hline & $\overline{\text { OR }}$ & $\mathbf{9 5} \% \mathrm{CI}$ & $P$ value & $\overline{\mathbf{O R}}$ & $\mathbf{9 5} \% \mathrm{CI}$ & $P$ value & $\overline{\mathbf{O R}}$ & $\mathbf{9 5} \% \mathrm{CI}$ & $P$ value \\
\hline EQ-5D anxiety/depression issue $(>1)$ & 2.04 & $0.97-4.26$ & .06 & 2.76 & $1.20-6.33$ & .02 & 4.41 & $1.51-12.92$ & $<.01$ \\
\hline PHQ-9 status (depressed) & 1.19 & $0.57-2.49$ & .60 & 1.40 & $0.62-3.19$ & .40 & 1.80 & $0.63-5.10$ & .30 \\
\hline AUDIT-C & 0.54 & $0.24-1.21$ & .10 & 0.55 & $0.23-1.34$ & .20 & 0.64 & $0.22-1.86$ & .40 \\
\hline
\end{tabular}

OR, Odds ratio; $C I$, confidence interval; EQ-5D, EuroQoL-5D; PHQ-9, Patient Health Questionnaire 9; AUDIT-C, Alcohol Use Disorders Identification Test Consumption *Model adjusts for age, sex, euroSCORE II. † $\nmid$ Model adjusts for age, sex, euroSCORE II, and baseline PHQ-9. 
and delirium rates in 734 nonenrolled patients during the study period. The nonenrolled cohort was composed of patients who required emergency surgery, did not meet eligibility criteria, were not seen in a preoperative clinic, did not consent, or were lost to follow-up. We are reassured that the rates of the postoperative delirium were similar in the nonenrolled patients $(23.3 \%$ vs $22.3 \%$ in the enrolled cohort, $P=.78)$ (Table E4).

Despite these limitations, we believe our study has important implications in characterizing the interrelationship between postoperative delirium and both cognitive impairment and affective functioning in the setting of cardiac surgery. The clinical relevance of these data is 2 -fold: First, given that postoperative delirium is associated with an increased risk of anxiety and depression at 6 to 9 months after surgery, care providers must be aware of the utility of screening for mood disorders in both the preoperative and postoperative patient. The identification of altered affective function in this population is a first step in the clinical pathway of connecting patients with appropriate treatment and rehabilitation to optimize outcomes. Second, with the identification of a number of risk factors for the development of delirium, it may facilitate improved prediction of postoperative delirium. Identifying those individuals at high risk for delirium is an important component to prevention. The current literature suggests that current efforts using a multipronged approach to prevention is more efficacious than treatment strategies after the development of delirium. ${ }^{32}$

\section{CONCLUSIONS}

In this prospective study of patients undergoing cardiac surgery, postoperative delirium was found to be a common neurologic complication and places patients at increased risk for anxiety and depression at 6 to 9 months after surgery. Further research is needed to understand the longerterm impact of these findings and to evaluate the utility of screening programs for affective disorders in those individuals who develop delirium in the postoperative period to optimize recovery.

\section{Conflict of Interest Statement}

Dr Arora has received an unrestricted educational grant from Pfizer Canada Inc, and honoraria from Mallinkrodt Pharmaceuticals for work unrelated to this article. All other authors have nothing to disclose with regard to commercial support.

\section{References}

1. Inouye SK, Westendorp RG, Saczynski JS. Delirium in elderly people. Lancet. 2014;383:911-22.

2. Arenson BG, MacDonald LA, Grocott HP, Hiebert BM, Arora RC. Effect of intensive care unit environment on in-hospital delirium after cardiac surgery. $J$ Thorac Cardiovasc Surg. 2013;146:172-8.
3. Koster S, Oosterveld FG, Hensens AG, Wijma A, van der Palen J. Delirium after cardiac surgery and predictive validity of a risk checklist. Ann Thorac Surg. 2008; $86: 1883-7$.

4. Gottesman RF, Grega MA, Bailey MM, Pham LD, Zeger SL, Baumgartner WA, et al. Delirium after coronary artery bypass graft surgery and late mortality. Ann Neurol. 2010;67:338-44.

5. Witlox J, Eurelings LS, de Jonghe JF, Kalisvaart KJ, Eikelenboom P, van Gool WA. Delirium in elderly patients and the risk of postdischarge mortality, institutionalization, and dementia: a meta-analysis. JAMA. 2010;304: 443-51.

6. Leslie DL, Marcantonio ER, Zhang Y, Leo-Summers L, Inouye SK. One-year health care costs associated with delirium in the elderly population. Arch Intern Med. 2008;168:27-32.

7. Deiner S, Silverstein JH. Postoperative delirium and cognitive dysfunction. $\mathrm{Br} \mathrm{J}$ Anaesth. 2009;103:i41-6.

8. Liang C-K, Chu C-L, Chou M-Y, Lin Y-T, Lu T, Hsu C-J, et al. Interrelationship of postoperative delirium and cognitive impairment and their impact on the functional status in older patients undergoing orthopaedic surgery: a prospective cohort study. PLoS One. 2014;9:e110339.

9. Koster S, Hensens AG, van der Palen J. The long-term cognitive and functional outcomes of postoperative delirium after cardiac surgery. Ann Thorac Surg. 2009;87:1469-74.

10. Saczynski JS, Marcantonio ER, Quach L, Fong TG, Gross A, Inouye SK, et al. Cognitive trajectories after postoperative delirium. N Engl J Med. 2012;367: 30-9.

11. Koster S, Hensens AG, Schuurmans MJ, van der Palen J. Consequences of delirium after cardiac operations. Ann Thorac Surg. 2012;93:705-11.

12. Nashef SA, Roques F, Sharples LD, Nilsson J, Smith C, Goldstone AR, et al. EuroSCORE II. Eur J Cardiothorac Surg. 2012;41:734-45.

13. Nasreddine ZS, Phillips NA, Bédirian V, Charbonneau S, Whitehead V, Collin I, et al. The Montreal Cognitive Assessment, MoCA: a brief screening tool for mild cognitive impairment. J Am Geriatr Soc. 2005;53:695-9.

14. Kroenke K, Spitzer RL, Williams JBW. The PHQ-9: validity of a brief depression severity measure. J Gen Intern Med. 2001;16:606-13.

15. Ely EW, Margolin R, Francis J, May L, Truman B, Dittus R, et al. Evaluation of delirium in critically ill patients: validation of the Confusion Assessment Method for the Intensive Care Unit (CAM-ICU). Crit Care Med. 2001;29:1370-9.

16. Inouye SK, van Dyck CH, Alessi CA, Balkin S, Siegal AP, Horwitz RI. Clarifying confusion: the confusion assessment method: a new method for detection of delirium. Ann Intern Med. 1990;113:941-8.

17. Radtke FM, Heymann A, Franck M, Maechler F, Drews T, Luetz A, et al. How to implement monitoring tools for sedation, pain and delirium in the intensive care unit: an experimental cohort study. Intensive Care Med. 2012;38:1974-81.

18. Devlin JW, Marquis F, Riker RR, Robbins T, Garpestad E, Fong JJ, et al. Combined didactic and scenario-based education improves the ability of intensive care unit staff to recognize delirium at the bedside. Crit Care. 2008;12:R19.

19. Johnson JA, Coons SJ. Comparison of the EQ-5D and SF-12 in an adult US sample. Qual Life Res. 1998; 7:155-66.

20. Janssen MF, Pickard AS, Golicki D, Gudex C, Niewada M, Scalone L, et al. Measurement properties of the EQ-5D-5L compared to the EQ-5D-3L across eight patient groups: a multi-country study. Qual Life Res. 2013;22:1717-27.

21. Rubinsky AD, Dawson DA, Williams EC, Kivlahan DR, Bradley KA. AUDIT-C scores as a scaled marker of mean daily drinking, alcohol use disorder severity, and probability of alcohol dependence in a U.S. general population sample of drinkers. Alcohol Clin Exp Res. 2013;37:1380-90.

22. Buschke H, Kuslansky G, Katz M, Stewart WF, Sliwinski MJ, Eckholdt HM, et al. Screening for dementia with the memory impairment screen. Neurology. 1999;52:231-8.

23. Kuslansky G, Buschke H, Katz M, Sliwinski M, Lipton RB. Screening for Alzheimer's disease: the memory impairment screen versus the conventional threeword memory test. J Am Geriatr Soc. 2002;50:1086-91.

24. Ting SK, Hameed S, Earnest A, Tan EK. Dissociative semantic breakdown in Alzheimer's disease: evidence from multiple category fluency test. Clin Neurol Neurosurg. 2013;115:1049-51.

25. Speidel H. Psychiatric issues: simple frequencies pre- and postoperatively. In: Willner AE, Rodewald G, eds. Impact of Cardiac Surgery on the Quality of Life: Neurologic and Psychologic Aspects. 1st ed. New York: Springer US; 1990:27-37.

26. Horne D, Kehler S, Kaoukis G, Hiebert B, Garcia E, Duhamel TA, et al. Depression before and after cardiac surgery: do all patients respond the same? J Thorac Cardiovasc Surg. 2013;145:1400-6. 
27. Bucerius J, Gummert JF, Borger MA, Walther T, Doll N, Falk V, et al. Predictors of delirium after cardiac surgery delirium: effect of beating-heart (off-pump) surgery. J Thorac Cardiovasc Surg. 2004;127:57-64.

28. Parikh SS, Chung F. Postoperative delirium in the elderly. Anesth Analg. 1995; 80:1223-32.

29. Nevin M, Colchester AC, Adams S, Pepper JR. Prediction of neurologic damage after cardiopulmonary bypass surgery: use of the cerebral function analysing monitor. Anaesthesia. 1989;44:725-9.

30. Fine TH, Contractor AA, Tamburrino M, Elhai JD, Prescott MR, Cohen GH, et al, Validation of the telephone-administered PHQ-9 against the in-person administered SCID-I major depression module. J Affect Disord. 2013;150:1001-7.
31. McPhail S, Lane P, Russell T, Brauer SG, Urry S, Jasiewicz J, et al. Telephone reliability of the Frenchay Activity Index and EQ-5D amongst older adults Health Qual Life Outcomes. 2009;7:48.

32. Milisen K, Lemiengre J, Braes T, Foreman MD. Multicomponent intervention strategies for managing delirium in hospitalized older people: systematic review. J Adv Nurs. 2005;52:79-90.

Key Words: cardiac surgery, cognition, comorbidity, mood, postoperative delirium

Readers who found these articles interesting may also like to read the following papers found in recent and future issues of our sister publications, Seminars in Thoracic and Cardiovascular Surgery and Operative Techniques in Thoracic and Cardiovascular Surgery!

\section{Acquired: Perioperative Management}

ORIGINAL SUBMISSION: Early Outcomes with Rapid-Deployment Versus Stented Biological Valves: a Propensity-Match Analysis. Anthony Nguyen. Semin Thoracic Surg 2017: In press.

ORIGINAL SUBMISSION: Randomized Trial of Carnitine for the Prevention of Perioperative Atrial Fibrillation. Farzaneh Dastan. Semin Thoracic Surg 2017: In press.

ORIGINAL SUBMISSION: Carbon Dioxide Insufflation during Cardiac Surgery. Meta-analysis of Randomized Controlled Trials. Umberto Benedetto. Semin Thoracic Surg 2017: In press.

ORIGINAL SUBMISSION: The Effects of Steroids on Coagulation Dysfunction Induced by Cardiopulmonary Bypass: A Steroids in Cardiac Surgery (SIRS) Trial Substudy. Domenico Paparella. Semin Thoracic Surg 2017: 35-44.

Editorial Commentary: Steroids and Cardiopulmonary Bypass: A Never-ending Story. Thierry Carrel. Semin Thoracic Surg 2017: 45-46. 
TABLE E1. Sensitivity analysis-excluding patients with preoperative depression

Association between postoperative delirium (CAM+) and cognition screening tools 6 months after cardiac surgery (excluding preoperative depressed patients)

\begin{tabular}{|c|c|c|c|c|c|c|c|c|c|}
\hline \multirow[b]{2}{*}{ Variable } & \multicolumn{3}{|c|}{ Unadjusted model } & \multicolumn{3}{|c|}{ Adjusted model* ${ }^{*}$} & \multicolumn{3}{|c|}{ Adjusted Model ${ }^{\dagger}$} \\
\hline & OR & $95 \% \mathrm{CI}$ & $P$ value & OR & $95 \% \mathrm{CI}$ & $P$ value & OR & $95 \% \mathrm{CI}$ & $P$ value \\
\hline MIS cognitive impairment & 3.66 & $1.54-8.74$ & $<.01$ & 2.02 & $0.76-5.35$ & .16 & 1.50 & $0.45-4.96$ & .51 \\
\hline CFT cognitive impairment & 1.54 & $0.68-3.50$ & .30 & 1.20 & $0.48-2.98$ & .69 & 1.04 & $0.34-3.21$ & .95 \\
\hline
\end{tabular}

Association between postoperative delirium (CAM+) and mood screening tools 6 months after cardiac surgery (excluding preoperative depressed patients)

\begin{tabular}{lccccccccc}
\hline $\begin{array}{l}\text { EQ-5D anxiety/depression } \\
\text { issue }(>1)\end{array}$ & 2.16 & $0.98-4.79$ & .06 & 3.12 & $1.22-7.98$ & .02 & 5.80 & $1.66-20.23$ & $<.01$ \\
PHQ-9 status (depressed) & 1.14 & $0.50-2.60$ & .76 & 1.40 & $0.54-3.62$ & .49 & 1.72 & $0.50-5.88$ & .39 \\
AUDIT-C & 0.78 & $0.34-1.81$ & .57 & 0.77 & $0.30-1.97$ & .58 & 1.18 & $0.37-3.78$ & .79 \\
\hline
\end{tabular}

CAM, Confusion Assessment Method; OR, odds ratio; $C I$, confidence interval; $M I S$, Memory Impairment Screen; $C F T$, Category Fluency Test; $E Q-5 D$, EuroQoL-5D; PHQ-9, Patient Health Questionnaire 9; AUDIT-C, Alcohol Use Disorders Identification Test Consumption. *Model adjusts for age, sex, and euroSCORE II. †Model adjusts for age, sex, euroSCORE II, and baseline MoCA. Model adjusts for age, sex, euroSCORE II, and baseline PHQ-9.

TABLE E2. Sensitivity analysis-with addition of covariate for time between surgery and contact

\begin{tabular}{|c|c|c|c|c|c|c|c|c|c|}
\hline \multicolumn{10}{|c|}{ Association between postoperative delirium $(\mathrm{CAM}+)$ and cognition screening tools 6 months after cardiac surgery } \\
\hline \multirow[b]{2}{*}{ Variable } & \multicolumn{3}{|c|}{ Unadjusted model } & \multicolumn{3}{|c|}{ Adjusted model ${ }^{*}$} & \multicolumn{3}{|c|}{ Adjusted model ${ }^{\dagger}$} \\
\hline & OR & $95 \%$ CI & $P$ value & OR & $95 \% \mathrm{CI}$ & $P$ value & OR & $95 \% \mathrm{CI}$ & $P$ value \\
\hline MIS cognitive impairment & 3.15 & $1.40-7.09$ & $<.01$ & 1.71 & $0.67-4.36$ & .26 & 1.03 & $0.33-3.20$ & .96 \\
\hline CFT cognitive impairment & 1.60 & $0.73-3.50$ & .20 & 1.38 & $0.58-3.28$ & .47 & 1.15 & $0.41-3.21$ & .79 \\
\hline \multicolumn{10}{|c|}{ Association between postoperative delirium $(\mathrm{CAM}+)$ and mood screening tools 6 months after cardiac surgery } \\
\hline $\begin{array}{l}\text { EQ-5D Anxiety/depression } \\
\text { issue }(>1)\end{array}$ & 2.04 & $0.97-4.26$ & .06 & 2.73 & $1.18-6.31$ & .02 & 4.48 & $1.51-13.32$ & $<.01$ \\
\hline PHQ-9 status (depressed) & 1.19 & $0.57-2.49$ & .60 & 1.38 & $0.61-3.15$ & .44 & 1.77 & $0.62-5.03$ & .28 \\
\hline AUDIT-C & 0.54 & $0.24-1.21$ & .10 & 0.53 & $0.22-1.30$ & .17 & 0.63 & $0.22-1.86$ & .41 \\
\hline
\end{tabular}

CAM, Confusion Assessment Method; OR, odds ratio; $C I$, confidence interval; $M I S$, Memory Impairment Screen; CFT, Category Fluency Test; EQ-5D, EuroQoL-5D; PHQ-9, Patient Health Questionnaire 9; AUDIT-C, Alcohol Use Disorders Identification Test Consumption. *Model adjusts for age, sex, and euroSCORE II. $\dagger$ Model adjusts for age, sex, euroSCORE II, and baseline MoCA. Model adjusts for age, sex, euroSCORE II, and baseline PHQ-9.

TABLE E3. Sensitivity analysis-adjust using propensity score instead of covariates

\begin{tabular}{|c|c|c|c|c|c|c|c|c|c|}
\hline \multirow[b]{2}{*}{ Variable } & \multicolumn{3}{|c|}{ Unadjusted model } & \multicolumn{3}{|c|}{ Adjusted model* } & \multicolumn{3}{|c|}{ Adjusted model $\dagger$} \\
\hline & OR & $95 \%$ CI & $P$ value & OR & 95\% CI & $P$ value & $\mathbf{O R}$ & 95\% CI & $P$ value \\
\hline \multicolumn{10}{|c|}{ Association between postoperative delirium $(\mathrm{CAM}+)$ and cognition screening tools 6 months after cardiac surgery } \\
\hline MIS cognitive impairment & 3.15 & $1.40-7.09$ & $<.01$ & 2.37 & $0.99-5.64$ & .05 & 1.84 & $0.68-4.97$ & .23 \\
\hline CFT cognitive impairment & 1.60 & $0.73-3.50$ & .20 & 1.61 & $0.72-3.63$ & .25 & 1.75 & $0.65-4.72$ & .27 \\
\hline \multicolumn{10}{|c|}{ Association between postoperative delirium $(\mathrm{CAM}+)$ and mood screening tools 6 months after cardiac surgery } \\
\hline $\begin{array}{l}\text { EQ-5D anxiety/depression } \\
\text { issue }(>1)\end{array}$ & 2.04 & $0.97-4.26$ & .06 & 2.33 & $1.10-4.95$ & .03 & 2.38 & $1.00-5.67$ & .05 \\
\hline PHQ-9 status (depressed) & 1.19 & $0.57-2.49$ & .60 & 1.23 & $0.58-2.61$ & .59 & 1.09 & $0.45-2.61$ & .85 \\
\hline AUDIT-C & 0.54 & $0.24-1.21$ & .10 & 0.59 & $0.26-1.34$ & .21 & 0.52 & $0.20-1.37$ & .99 \\
\hline $\begin{array}{l}O R \text {, Odds ratio; } C I \text {, confidence } \\
\text { Patient Health Questionnaire } 9 ; \\
\text { ables: age, sex, education, race, } \\
\text { pulmonary disease, congestive } \\
\text { II. } † \text { Model adjusts for propensity } \\
\text { conditions (atrial fibrillation, car } \\
\text { infarction, diabetes), procedure }\end{array}$ & $A M, \mathrm{C}$ & $\begin{array}{l}\text { ion Assessme } \\
\text { e Disorders Id } \\
\text { one, previous } \\
\text { ufficiency, dia } \\
\text { ding the follo }\end{array}$ & $\begin{array}{l}\text { s, hypert } \\
\text { g variabl }\end{array}$ & emory & $\begin{array}{l}\text { infarction, di } \\
\text { ion, race, live }\end{array}$ & $T$, Catego & $\begin{array}{l}\text { ype, } \\
\text { previ }\end{array}$ & $\begin{array}{l}\text { ure urgency, } \\
\text { rdiac interve }\end{array}$ & preexisti \\
\hline
\end{tabular}


TABLE E4. Comparison between enrolled cohort and nonenrolled patients

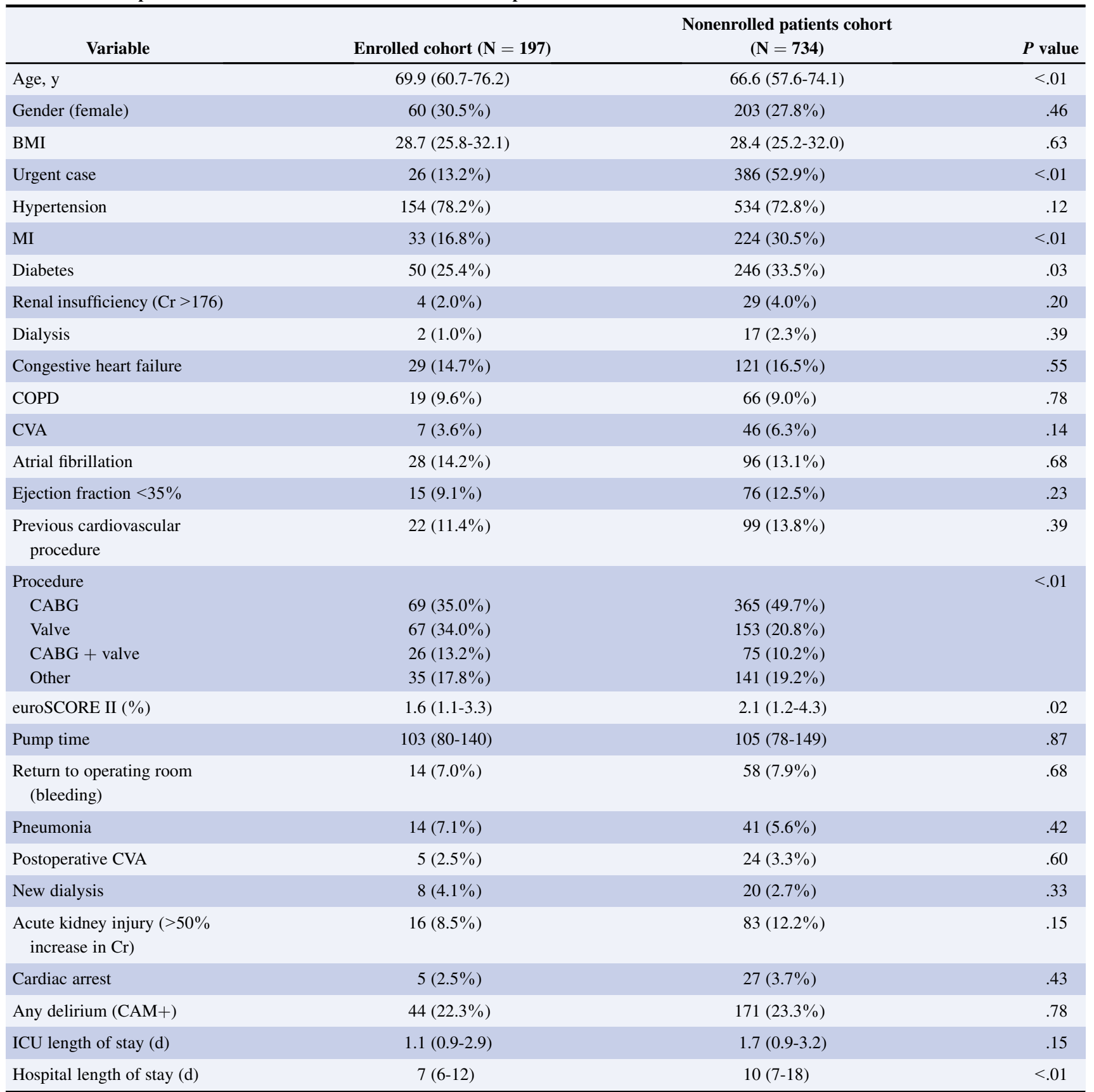

Continuous variables are expressed as median (interquartile range) and compared using Mann-Whitney test. Categoric variables are expressed as N (\%) and compared using chisquare test or Fisher exact test. BMI, Body mass index; $M I$, myocardial infarction; $C r$, creatinine; $C O P D$, chronic obstructive pulmonary disease; $C V A$, cardiovascular accident; $C A B G$, coronary artery bypass grafting; euroSCORE, European System for Cardiac Operative Risk Evaluation; CAM, Confusion Assessment Method; ICU, intensive care unit. 